\title{
Antagonists of the Anaphylactoid Reaction
}

\begin{tabular}{|l|l|}
\hline S.I. & Ankier \\
\hline G.B. & West \\
\hline
\end{tabular}

From the School of Pharmacy, University of London

Authors' address: Dr. R. . West and Dr. S.I. Ankier, Dept. of Pharmacology, School of Pharmacy, 29, Brunswick Square, London, W. C. 1 (England)

A single intraperitoneal or intravenous injection of fresh egg-white, dextran or dextrin into rats produces an acute inflammatory response called the anaphylactoid reaction. Dose-response curves of these active materials have now been determined. The threshold doses of the three agents by the intravenous route were respectively 6,2 , and 8 times less than those needed intraperitoneally. In other experiments, rats were treated twice daily with one of the agents intraperitoneally and then on the fifth day they received either intraperitoneally or intravenously one of the three materials. Almost complete refraction was observed in all those rats which were challenged intraperitoneally, whilst in those rats which were challenged intravenously the eggwhite and dextran responses were reduced but the dextrin response was unaltered.

Whereas mepyramine given intravenously did not modify any of the responses, cyproheptadine and bromo-lysergic acid diethyl-amide (two powerful anti-serotonin compounds) inhibited all three responses. Serotonin, and not histamine, therefore appears to be important in mediating the anaphylactoid reaction.

In the later studies, D-glucose, D-mannose, $\mathrm{N}$-acetylglucosa-mine and L-ascorbic acid were found to be effective in inhibiting the anaphylactoid reaction produced by fresh egg-white, dextran or dextrin. It thus seems that the reaction is related to carbohydrate metabolism; it is possible that rat mast cells have receptors for dextran and related carbohydrates and these have the characteristics of the glucose carrier system as considered in the field in sugar transport. 